\title{
Enhancing Materials and Device Analysis Capability in the SEM and FIB-SEM by using a Nanomanipulator
}

\author{
Matthew Hiscock ${ }^{1}$, Christian Lang ${ }^{1}$, Peter Statham ${ }^{1}$, Frank Bauer ${ }^{2}$ and Cheryl Hartfield ${ }^{3}$ \\ 1. Oxford Instruments Nanoanalysis, High Wycombe, United Kingdom \\ 2. Oxford Instruments Nanoanalysis, Wiesbaden, Germany \\ 3. Oxford Instruments Nanoanalysis, Dallas, United States.
}

Nanomanipulators, whilst commonly used to lift out TEM samples prepared in the FIB, have a number of other potential applications and can aid in the chemical and crystallographic analysis of samples. Here, we discuss some of these alternative uses of nanomanipulators and show how they enable the EDS and EBSD analysis of thin samples in the FIB-SEM as well as serve as electrical probes and enable charge dissipation on uncoated samples.

Figure 1 (a) shows an EDS map of a gunshot residue particle picked up with an OP400 nanomanipulator from a sample stub in the FIB. Depending on how strongly particles are attached to the substrate, the attachment to the nanomanipulator may require welding in a FIB or electrostatic attachment to the tip may be sufficient. The advantage of analyzing the particle when it is attached to the tip is that it can be easily reoriented using the tip rotation function whereas on the substrate any reorientation is limited by the available stage movements and shadowing of any particle close by. If the sample analysis is performed in a dual-beam FIB then the ion beam can be used to investigate the inside of the particle using both EDS and EBSD (Figure 1(b)) or thin the sample further until it becomes electron transparent. This enables the analysis of the sample in transmission, with high spatial resolution using both TKD for crystallographic information and EDS for compositional information.

When using an OP400, a voltage can be applied to the tip to perform voltage contrast measurements and with additional hardware the system can be extended to perform EBIC and EBAC measurements. While this allows for enhanced electrical characterization of samples and can be used to identify defects such as the COX breakdown in a capacitor shown in Figure 2, in its simplest case the probe can be used to dissipate undesirable charge build-up from the electron beam. For insulating samples, charge induced by the electron beam builds up very quickly and the resulting fields lead to a deflection and distortion of both the electron beam and any secondary or backscattered electrons (Figure 3(a)), resulting in distortions or contrast changes in the electron image. The build-up of an electric field also decelerates the beam, reducing the landing energy of the electrons resulting in changes in X-ray spectra $\&$ maps (Figure 3(b \& c)). This was confirmed by simulating spectra at different voltages (Figure $3(d)$ ). Charge can be dissipated by coating the sample surface with a conductive element and then grounding the sample surface (e.g. by forming a conductive connection to the SEM stage). However, especially for high resolution analytical work, metallic elements are usually not desirable for the coating layer as they can lead to significant X-ray absorption especially of lighter elements in the substrate. Any deposition of a surface layer will also reduce the quality of electron backscattered diffraction patterns and therefore limit the speed and quality of any EBSD data that can be obtained from the sample. By dissipating charge locally as it builds up through an electrically grounded probe we show that any negative effects can be significantly reduced with the possibility to obtain high quality analytical data from uncoated, insulating samples, especially at low $\mathrm{kV}$. The area over which charge can be dissipated will depend on the sample surface and imaging conditions. 

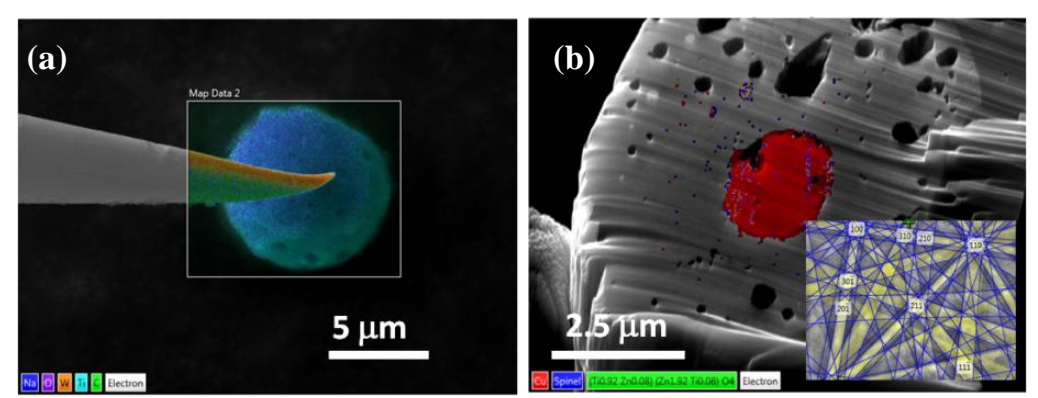

Figure 1. (a) EDS map of a GSR particle attached to the tip of a nanomanipulator. (b) a particle held on a nanomanipulator cut open in the FIB. The EDS map shows an embedded $\mathrm{Cu}$ particle and its crystallinity is confirmed using EBSD (see inset).

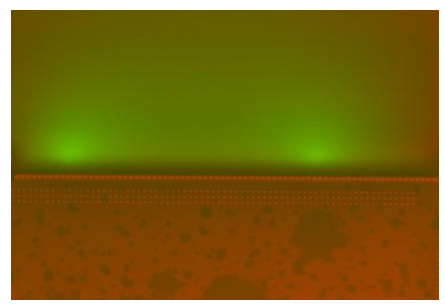

Figure 2. Mixed SE/ËBIC image showing two COX breakdowns (bright green areas) in a failed capacitor.
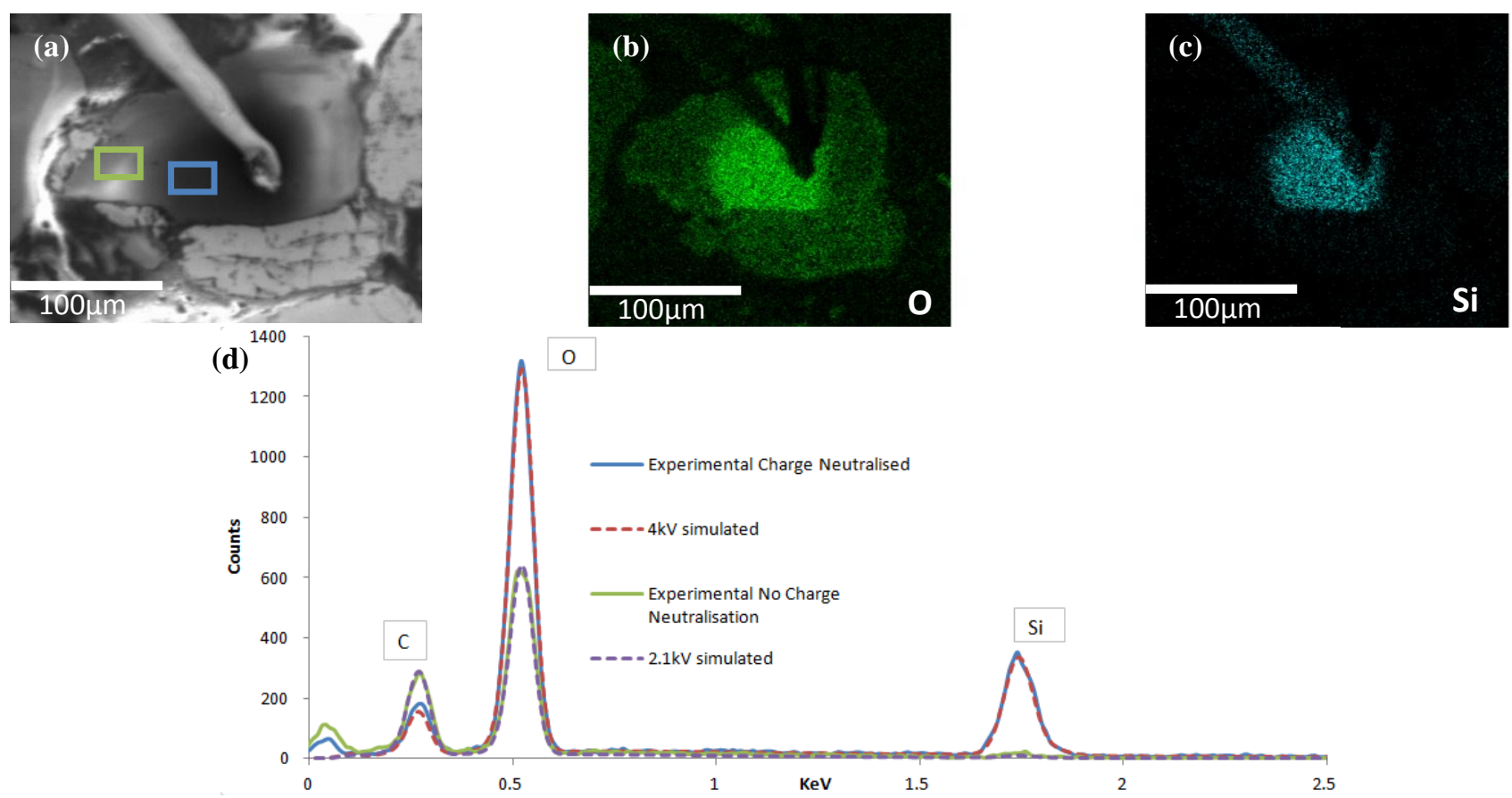

Figure 3. (a) Electron image (s.e.) and (b,c) X-ray maps at $4 \mathrm{kV}$ showing a charging $\mathrm{SiO}_{2}$ sample grounded with a nanomanipulator probe tip - the neutralized area is dark in the electron image with $\mathrm{X}^{-}$ ray maps in the region enhanced. (d) Comparison of spectra acquired from the regions marked in (a) (color key matches) - the charge neutralized experimental spectrum is shown to closely match a $4 \mathrm{kV}$ simulated spectrum and the non-charge neutralized spectrum is shown to match a $2.1 \mathrm{kV}$ spectrum indicating that the build-up of surface charge has the effect of decelerating the beam and reducing the landing voltage. 